\section{Fibras na dieta: tendências atuais e benefícios à saúde na síndrome metabólica e no diabetes melito tipo 2}

\author{
Dietary fibers: current trends and health benefits in \\ the metabolic syndrome and type 2 diabetes
}

Vanessa D. de Mello', David E. Laaksonen ${ }^{2,3}$

\section{RESUMO}

As fibras dietéticas parecem contribuir tanto na prevenção quanto no tratamento do diabetes melito tipo 2 (DMT2). Em estudos epidemiológicos a ingestão de fibras insolúveis, e não de fibras solúveis, tem sido inversamente associada à incidência do DMT2. Por outro lado, em estudos pós-prandiais, refeições contendo quantidades suficientes de $\beta$-glucano, psyllium, ou goma-guar diminuíram as respostas da insulina e da glicose, tanto em indivíduos saudáveis como em pacientes com DMT2. Dietas enriquecidas com quantidade suficiente de fibras solúveis também parecem melhorar o controle glicêmico de uma forma geral no DMT2. As fibras insolúveis têm pouco efeito sobre as respostas pós-prandiais de insulina e glicose. As fibras da dieta aumentam a saciedade. Em alguns estudos, as fibras solúveis têm se associado com um menor aumento de peso corporal ao longo do tempo. Evidências limitadas, a partir de estudos transversais, sugerem uma associação inversa entre o consumo de fibras dos cereais e de grãos integrais e a prevalência de síndrome metabólica. Apesar da escassez de dados sobre estudos de mais longo prazo que foquem especificamente em fibras dietéticas, seguir a recomendação atual de $25 \mathrm{~g}$ de fibras ao dia, a partir de uma dieta rica em grãos integrais, frutas e legumes, provavelmente diminuirá o risco para a obesidade, síndrome metabólica e DMT2. Arq Bras Endocrinol Metab. 2009;53(5):509-18.

Descritores

Fibras na dieta; cereais; insulina; glicose; diabetes melito tipo 2; síndrome metabólica

\section{ABSTRACT}

Dietary fiber may contribute to both the prevention and treatment of type 2 diabetes mellitus (T2DM). In epidemiological studies the intake of insoluble fiber, but not the intake of soluble fiber, has been inversely associated with the incidence of T2DM. In contrast, in postprandial studies, meals containing sufficiently quantities of $\beta$-glucan, psyllium, or guar gum have decreased insulin and glucose responses in both healthy individuals and patients with T2DM. Diets enriched sufficiently in soluble fiber may also improve overall glycemic control in T2DM. Insoluble fiber has little effect on postprandial insulin and glucose responses. Fiber increases satiety. In some studies, insoluble fiber has been associated with less weight gain over time. Limited cross-sectional evidence suggests an inverse relationship between intake of cereal fiber and whole-grains and the prevalence of the metabolic syndrome. Although long-term data from trials focusing on specifically dietary fiber are lacking, meeting current recommendations for a minimum fiber intake of $25 \mathrm{~g} / \mathrm{d}$ based on a diet rich in whole grains, fruits and legumes will probably decrease the risk of obesity, the metabolic syndrome and T2DM. Arq Bras Endocrinol Metab. 2009;53(5):509-18.

Key words

Dietary fiber; cereals, insulin; glucose; type 2 diabetes mellitus; metabolic syndrome
${ }^{1}$ School of Public Health and Clinical Nutrition, Department of Clinical Nutrition, University of Kuopio, Kuopio, Finland ${ }^{2}$ Institute of Clinical Medicine, Internal Medicine, Kuopio University Hospital, Kuopio, Finland ${ }^{3}$ Institute of Biomedicine, Physiology, University of Kuopio, Kuopio, Finland

Correspondência para: Vanessa D. de Mello

School of Public Health and Clinical Nutrition - Department of Clinical Nutrition, University of Kuopio P.O. Box 1627, FIN-70211 - Kuopio, Finland

vanessa.ferreirademello@uku.fi

Recebido em 28/Fev/2009 Aceito em 5/Jun/2009 
$\mathrm{O}$ s efeitos fisiológicos das fibras da dieta, assim como de suas propriedades físicas inerentes ao alimento, têm sido foco de atenção na prevenção do diabetes melito tipo 2 (DMT2). O DMT2, definido como uma desordem metabólica de múltiplas etiologias, caracteriza-se por um estado de crônica hiperglicemia com consequentes distúrbios no metabolismo de carboidratos, lipídeos e proteínas, os quais resultam de defeitos na secreção e geralmente na ação do hormônio insulina. Em razão do aumento da prevalência de obesidade, da inatividade física e de fatores dietéticos, o DMT2 tem sido considerado uma das principais epidemias dos séculos XX e XXI. O DM é a quarta causa de morte no mundo e estima-se que no ano 2030 a sua prevalência mundial seja superior a 360 milhões de casos (http:// www.who.int/diabetes/facts/world_figures/en acessado em janeiro de 2009). Ademais, os custos de tratamento do DMT2 para a saúde pública têm aumentado de uma maneira escalar em função da elevação expressiva das complicações vasculares. Evidências convincentes mostram que o tratamento da hiperglicemia reduz o risco das complicações microvasculares do DM e que o tratamento dos fatores de risco cardiovascular previne as complicações macrovasculares da doença (1). Recentemente, de relevante importância, está o fato de que estudos de intervenção têm mostrado que o DMT2 pode ser prevenido a partir de mudanças no estilo de vida. Essas mudanças incluem, além de moderada perda de peso e aumento da atividade física, também a diminuição do consumo de gorduras, especialmente as compostas por ácidos graxos saturados e o aumento do consumo de fibras alimentares $(2,3)$.

Resultados de estudos epidemiológicos sugerem que os carboidratos com grande quantidade de fibras e baixo índice glicêmico ou carga glicêmica e com grande quantidade de fibras na sua composição diminuem o risco de DMT2. Estudos de intervenção mostram que tanto o índice glicêmico do alimento quanto a sua quantidade de fibra solúvel resultam em efeitos favoráveis sobre as respostas glicêmica e insulínica pós-prandiais em pacientes com e sem DM. Em pacientes com DMT2, porém, estudos mostram que esse benefício sobre o controle glicêmico ocorre também a longo prazo.

O presente artigo tem como objetivo revisar brevemente o papel das fibras da dieta em relação ao metabolismo da glicose e insulina, à patogênese do DMT2 e aos distúrbios metabólicos que geralmente precedem o seu aparecimento, incluindo a síndrome metabólica. Será mais detalhada, no entanto, a abordagem sobre as evidências do papel das fibras dietéticas na redução do risco de obesidade, da síndrome metabólica e do DMT2, assim como o seu benefício no tratamento dessas condições. De forma breve, também será abordado o tema em relação aos benefícios das fibras nas áreas de nutrigenômica e metabolômica, caracterizando os avanços científicos nas metodologias. Outros componentes e fatores presentes nas fontes dos alimentos ricos em fibras, como o índice glicêmico e a carga glicêmica, e outros macronutrientes não serão abordados de forma abrangente nesta revisão.

\section{PATOGÊNESE DA SÍNDROME METABÓLICA E DO DIABETES MELITO TIPO 2}

A patogênese da síndrome metabólica e do DMT2 não é ainda muito bem elucidada. A discussão sobre a patogênese da síndrome metabólica e do DMT2 vai além dos objetivos desta revisão. No entanto, é evidente que ambos estados ocorrem como o resultado da interação entre fatores genéticos e ambientais, e são ambos poligenéticos por natureza. Como consequência, o fenótipo que caracteriza tanto o DMT2 quanto a síndrome metabólica varia. Para o DMT2, esse problema é de menor magnitude, pois sua definição depende somente de valores estabelecidos para a glicose de jejum ou pósprandial. Já na síndrome metabólica, a variabilidade fenotípica é maior, pois baseia-se em vários componentes, o que dificulta uma definição que seja amplamente aceita. Ademais, a relevância clínica das várias definições existentes para a síndrome metabólica está ainda em debate.

Várias definições têm sido propostas para a classificação da síndrome metabólica. Talvez a mais amplamente utilizada para caracterizar essa síndrome seja aquela definida pelo National Cholesterol Education Program Expert Panel (NCEP ATP III (4). A síndrome metabólica definida pelo NCEP é muito comum e está presente em cerca de $24 \%$ dos indivíduos nos Estados Unidos, com proporções um pouco menores na maioria dos países europeus. Dados sobre a sua prevalência no Brasil ainda não existem. A definição da NCEP engloba a presença de três de cinco componentes: obesidade abdominal, hiperglicemia de jejum, pressão arterial elevada, triglicerídeos elevados, HDL reduzido. A definição de síndrome metabólica mais recente do International Diabetes Federation (IDF) também é muito utilizada. $\mathrm{O}$ IDF condiciona a classificação à presença de gordura abdominal alterada (5). A definição da IDF de síndro- 
me metabólica requer a presença de obesidade central, definida pela circunferência abdominal, além de outros dois dos quatro seguintes fatores: elevação de triglicerídeos ou tratamento para tal; HDL reduzido ou tratamento para elevá-lo; hipertensão arterial ou tratamento anti-hipertensivo; hiperglicemia de jejum ou diabetes previamente diagnosticado.

De qualquer forma classificada, a síndrome metabólica constitui uma importante ameaça à saúde pública. Isso ocorre tanto em função da sua associação com o aumento de 5 a 10 vezes no risco de desenvolver DMT2 e com o aumento de 2 a 3 vezes no risco para a doença cardiovascular (6-8). Fatores de risco para o desenvolvimento da doença cardiovascular como a dislipidemia, a hipertensão, a disfunção endotelial, a inflamação, a hipercoagulação e as alterações na fibrinólise, a obesidade e o metabolismo alterado da insulina e glicose fazem parte da síndrome metabólica ou são fortemente associados a ela.

À medida que a síndrome metabólica vai tornandose mais severa, uma ação recíproca entre a suscetibilidade genética, a resistência à insulina e os padrões dietéticos pode levar à falência das células beta $(\beta)$ do pâncreas e a anormalidades na secreção da insulina. Com o declínio da função das células $\beta$, a intolerância à glicose se desenvolve (9). Neste ponto, a primeira fase da secreção de insulina e a secreção pulsátil de insulina, ambas essenciais para a tolerância normal à glicose, estão prejudicadas, acarretando uma exagerada longa duração da segunda fase de secreção de insulina. Aproximadamente, de $5 \%$ a $10 \%$ das pessoas com intolerância à glicose tornam-se diabéticas a cada ano (10). No entanto, o DMT2 é uma entidade clínica heterogênica, e por isso nem todos os pacientes apresentam os componentes da síndrome metabólica; aproximadamente de $10 \%$ a $20 \%$ têm deficiência da secreção de insulina como fator que mais contribui para a hiperglicemia.

\section{DEFINIÇÃO DE FIBRAS E SUAS PRINCIPAIS FONTES NA DIETA}

O que denominamos de fibra da dieta é uma definição nutricional da parte não digerível do alimento vegetal, a qual resiste à digestão e absorção intestinal, porém com fermentação completa ou parcial no intestino grosso. Apesar de discussões entre grupos de estudiosos, a maioria deles concorda que oligossacarídeos, celulose, hemicelulose, pectinas, gomas, lignina, polissacarídeo indigeríveis e não amilosos, além de ceras e outras substâncias inerentes às plantas, devem ser classificados como fibras dietéticas (11). No entanto, a sua definição é complexa e de evolução contínua. Mais recentemente, a classificação da Comissão em Nutrição e Alimentos para Usos Especiais na Dieta (CODEX) refere que as fibras dietéticas são "polímeros de carboidratos com dez ou mais unidades monoméricas, as quais não são hidrolisadas por enzimas endógenas no intestino de seres humanos e que pertencem às seguintes categorias: 1) polímeros de carboidratos comestíveis inerentes aos alimentos que são consumidos; 2) polímeros de carboidratos que tenham sido obtidos a partir de matéria-prima alimentar por meio de procedimentos enzimáticos, físicos ou químicos, os quais tenham mostrado algum efeito fisiológico benéfico à saúde através de meios científicos aceitos pelas autoridades competentes, ou 3 ) polímeros de carboidratos sintéticos que tenham mostrado algum efeito fisiológico benéfico à saúde através de meios científicos aceitos pelas autoridades competentes" (12).

Com base na sua solubilidade, as fibras provenientes da dieta podem ser classificadas em fibras solúveis e insolúveis. As fibras solúveis incluem as pectinas, gomas, mucilagens (como o psyllium, um polissacarídeo viscoso) e polissacarídeos de armazenagem. Entre as fibras insolúveis estão a celulose, as hemiceluloses e a lignina. A aveia é uma fonte muito rica em fibras solúveis, principalmente o seu farelo. As frutas e vegetais também são fontes de fibras solúveis, porém em menor quantidade, principalmente de pectinas. Os legumes (feijões, lentilha, ervilhas) e cereais são fontes de ambos os tipos de fibras, sendo que os cereais em geral têm na sua composição um maior teor de fibras insolúveis como no caso dos grãos integrais (cevada, farinha integral), o arroz e o centeio (13). No entanto, as fibras também podem ser obtidas por meio de suplementos, no caso, suplementos de fibras solúveis (psyllium, inulina, gomas).

A fim de auxiliar na prevenção do aparecimento de doenças crônicas relacionadas à dieta, a FAO/OMS recomenda o consumo de pelo menos $25 \mathrm{~g} / \mathrm{d}$ de fibras na dieta (14). No entanto, em muitos países a adesão a essa recomendação não é alcançada.

\section{Fibras da dieta na prevenção da obesidade e síndrome metabólica}

Mecanismos que diminuem o risco de obesidade e promovem a perda de peso são críticos na prevenção do DMT2. Estudos epidemiológicos sugerem que as fibras dos cereais e produtos à base de grãos integrais são capazes de prevenir a obesidade e o ganho de peso, além de contribuir na diminuição do risco para o desenvolvi- 
mento de DM (15). No entanto, os resultados obtidos a partir dos estudos de intervenção são conflitantes. Neste sentido, também existe atualmente certa controvérsia em relação ao papel das fibras solúveis na perda de peso.

\section{Estudos epidemiológicos}

Com base nos estudos epidemiológicos, a melhor evidência para o papel das fibras na prevenção da obesidade e síndrome metabólica aponta para as fibras insolúveis. Vários estudos revelam associação entre a presença de obesidade ou aumento de peso ao longo do tempo e um maior consumo energético na dieta e uma menor ingestão de alimentos ricos em fibras, como frutas e vegetais $(16,17)$. Em alguns estudos observacionais do tipo transversal, o consumo de fibra foi direta ou indiretamente associado a medidas de peso e gordura corporal, esta última com base na medida de três dobras cutâneas (18). No entanto, o tamanho amostral destes estudos impossibilita extrapolações desses resultados de uma maneira mais abrangente. Por outro lado, um estudo com desenho experimental do tipo ecológico com cerca de 12.700 homens de sete diferentes países (Seven Countries Study) mostrou que a ingestão de fibras na dieta e a atividade física relacionada ao trabalho são fatores determinantes da gordura corporal avaliada pela medida da dobra cutânea subescapular (19).

Já os estudos observacionais de caráter longitudinal mostram resultados mais sólidos. Um desses estudos, com caráter multicêntrico e populacional, em que quase 3.000 adultos jovens foram seguidos por 10 anos, o consumo de fibras na dieta se mostrou inversamente associado ao IMC em todos os diferentes níveis de consumo de gordura após ajustes para outros fatores de confusão (20). A partir de um maior detalhamento na avaliação da dieta, o estudo sobre a saúde das enfermeiras - Nurses' Health Study - mostrou que o ganho de peso ao longo dos 12 anos de seguimento do estudo foi inversamente proporcional ao consumo de fibras, alimentos à base de grãos integrais e positivamente associado com o consumo de alimentos à base de grãos refinados (15). Em um estudo conduzido em cerca de 27.000 homens entre 40 e 75 anos de idade também foi demonstrada associação inversa entre o aumento do consumo de grãos integrais e o ganho de peso ao longo dos oito anos de seguimento (21). Isso ocorreu de forma dose-dependente, pois, para cada $40 \mathrm{~g}$ diários a mais na dieta de grãos integrais derivados de qualquer alimento, o ganho de peso foi reduzido em $0,49 \mathrm{~kg}$. No entanto, os autores não atribuem somente às fibras a explicação para tal efeito benéfico. O consumo de fibras oriundo de frutas e cereais nesta população também foi inversamente associado ao ganho de peso. Mais recentemente, num estudo transversal de menor porte com cerca de 2.000 mulheres que fizeram parte da população do estudo National Health and Nutrition Examination Survey 1999-2000, foi avaliada a relação entre a ingestão de grãos integrais e o IMC. Os autores demonstraram que mulheres que costumavam consumir mais frequentemente grãos integrais tinham valores de IMC e circunferência da cintura menores, e menos predisposição para terem sobrepeso (22). Uma recente revisão sistemática sobre a ingestão de grãos integrais e adiposidade revelou que o consumo desses grãos estava associado a uma maior e menor ingestão de fibras e gorduras na dieta, a partir dos estudos selecionados ( 15 estudos observacionais que resultaram em cerca de 120.000 homens e mulheres com mais 13 anos de idade). Esta revisão mostrou que a ingestão de três porções de grãos integrais por dia se associou com menores valores de IMC e de obesidade central (23).

Em relação à manutenção de peso e obesidade, esses estudos demonstram que as fibras insolúveis, principalmente provenientes dos grãos integrais, possuem um papel benéfico. No entanto, os efeitos desses grãos integrais parecem não ser explicados somente pelo seu conteúdo de fibras, uma vez que o efeito fisiológico das fibras solúveis é que teria um papel na saciedade devido a sua maior viscosidade, a qual promove um atraso no esvaziamento gástrico, na absorção intestinal ou em ambos.

Resultados com base em estudos observacionais mostram uma associação negativa do consumo de fibras e síndrome metabólica. No entanto, em um desses estudos, o consumo das fibras derivadas dos cereais (cereais integrais), e não das fibras oriundas das frutas, vegetais e legumes, é que estava inversamente associado à prevalência de síndrome metabólica (24). O maior consumo de grãos integrais e de fibras dos cereais também estava associado a menores índices de resistência à insulina. Neste estudo participaram 2.834 indivíduos e a síndrome metabólica foi definida pelo critério do NCEP (4). Em outro estudo, porém com indivíduos com risco para desenvolvimento de DM, Ylonen e cols. avaliaram transversalmente cerca de 500 indivíduos. Os autores também observaram uma associação inversa entre o consumo total de fibras, tanto de fibras insolúveis como solúveis, e os índices de resistência insulínica (25). No entanto, não foram conduzidas análises mais detalhadas em relação às diferentes fontes de fibras na dieta, 
o que talvez tenha ocorrido pelo seu número limitado de participantes ou pelo não direcionamento adequado do recordatório alimentar utilizado para esses fins. Mesmo com número similar de participantes ( $\mathrm{n}=\mathbf{5 3 5}$ sendo 179 homense 356 mulheres), outros autores observaram que, em indivíduos que consumiam em média 2,9 porções de grãos integrais ao dia, a prevalência de síndrome metabólica foi menor (razão de chance: 0,46; 95\% IC: $0,27,0,79)$ do que em indivíduos que consumiam em média menos do que uma porção diária desses grãos (26). Como já mencionado, a heterogeneidade na classificação da própria síndrome metabólica por si só dificulta estudos epidemiológicos que relacionem o consumo de fibras e a sua prevalência ou risco de seu desenvolvimento ao longo do tempo. Poucos são os estudos neste tópico. Mais recentemente, porém, em pacientes com DMT2, a ingestão de fibras solúveis derivadas de grão integrais e de frutas associou-se inversamente com a síndrome metabólica, a qual foi definida pela IDF nesta população (27).

\section{Fibras da dieta no tratamento da obesidade e metabolismo da glicose e insulina}

\section{Estudos pós-prandiais}

Doses altas de $\beta$-glucano, um tipo de fibra solúvel presente em grandes quantidades no farelo da aveia, diminui as respostas pós-prandiais de insulina e glicose em indivíduos saudáveis $(28,29)$. O psyllium, prescrito em doses diárias de $5 \mathrm{~g}$ ou mais, também foi capaz de diminuir as concentrações de glicose e insulina pós-prandiais em indivíduos não diabéticos (30). Porém, uma dose de somente $2,3 \mathrm{~g}$ por dia (31) não resultou em efeito semelhante. Por outro lado, a goma-guar tem mostrado efeito benéfico na redução dos níveis pós-prandiais de insulina e da concentração de glicose em vários estudos em indivíduos não diabéticos (32, 33). Porém, apesar de manter o efeito benéfico nos níveis pós-prandiais de insulina, em alguns estudos não mostrou o mesmo efeito na redução das concentrações plasmáticas de glicose (34). Uma refeição rica em fibras, com base em cevada e com baixo índice glicêmico (índice glicêmico 53 ), quando consumida à noite como última refeição, foi capaz de melhorar a tolerância à glicose após o café da manhã, enquanto uma refeição consumida no mesmo horário, porém composta de macarrão (índice glicêmico 54), não teve efeito algum. Esses achados atentam para a importância do conteúdo de fibras na dieta para a obtenção de uma resposta satisfatória após a segunda refeição (35). Resultados semelhantes têm sido descritos quando se estudam as refeições de café da manhã e do almoço (36).

\section{Estudos de médio e longo prazos}

São poucos os estudos de médio e longo prazos (por exemplo, que tenham duração de semanas a meses) sobre os efeitos da aveia, psyllium e goma-guar no metabolismo da glicose e insulina em indivíduos não diabéticos. Em um estudo com 36 homens não diabéticos, com sobrepeso ou obesidade, e de meia-idade ou idosos, o consumo de farelo de aveia na dieta ( $14 \mathrm{~g}$ de fibra dietética, $5,5 \mathrm{~g}$ $\beta$-glucano) por 12 semanas melhorou a eficácia do metabolismo da glicose quando comparado à ingestão de cereal de trigo (37). Porém, não houve efeito algum na sensibilidade à insulina ou na resposta aguda da primeira fase da insulina à infusão de glicose, ambas analisadas pelo teste minimal model. Uma dieta à base de cevada e enriquecida de $\beta$-glucano (8-12 g/dia), administrada por 4 semanas, não resultou em efeito significativo na tolerância à glicose em um estudo do tipo cruzado conduzido em 18 homens hiperlipidêmicos e com certo grau de sobrepeso (38). $\mathrm{O}$ consumo de farelo de aveia $(100 \mathrm{~g} / \mathrm{dia})$ ou farelo de aveia enriquecido de $10,3 \mathrm{~g} /$ dia de $\beta$-glucano também não demonstrou efeito algum nas concentrações de glicose ou insulina plasmática em indivíduos hipercolesterolêmicos que participaram tanto de um ensaio clínico do tipo randomizado e cruzado $(\mathrm{n}=8,(39)$ ou de um estudo randomizado do tipo paralelo $(\mathrm{n}=52,(40)$, respectivamente. Da mesma maneira, a adição de $3,4 \mathrm{~g}$ de psyllium nas refeições, por um período de oito semanas, também não foi capaz de reduzir os níveis de glicose de jejum em dois ensaios clínicos randomizados e controlados conduzidos em 26 (41) e 75 (42) indivíduos hipercolesterolêmicos.

Poucos estudos em que se compararam diferentes tipos de carboidratos com diferentes tipos ou conteúdos de fibras foram conduzidos em indivíduos com síndrome metabólica. Um recente estudo, conduzido em 50 homens e mulheres, mostrou que 12 semanas de uma dieta hipocalórica com mais de três porções de grãos integrais por dia versus uma dieta hipocalórica, porém isenta de grãos integrais, foi capaz de diminuir o peso corporal, a gordura abdominal (circunferência da cintura) e a resposta inflamatória (concentração de proteína $\mathrm{C}$ reativa circulante) dos participantes do estudo (43). Outro estudo, de menor duração (6 semanas, $\mathrm{n}=11$ ), que utilizou um suplemento à base de arabinoxilano - uma fibra solúvel, demonstrou que a dieta suplementada com $15 \mathrm{~g}$ desse polímero foi capaz de diminuir a glicemia de jejum e os triglicerídeos séricos, porém sem efeito sobre os níveis de insulina (44).

Refeições com alto teor de fibras insolúveis não têm demonstrado resultados consistentes em relação ao seu 
possível benefício nas respostas pós-prandiais de glicose e insulina. A resposta insulínica a uma refeição com pão de centeio como tipo de pão que acompanha a refeição é mais baixa do que aquela com pão de farinha de trigo. Ademais, a resposta da primeira fase da secreção de insulina parece aumentar com o consumo da dieta que utiliza o pão de centeio em comparação ao pão de farinha de trigo. No entanto, essa diferença não é devido ao seu conteúdo de fibras. Outros fatores como a estrutura física do pão de centeio ou possivelmente outros componentes inerentes ao pão ou aos ingredientes do pão talvez expliquem o aparente efeito benéfico do pão de centeio no metabolismo dos glicídios e da insulina em relação ao pão de trigo $(45,46)$.

Em suma, as fibras insolúveis não possuem efeitos pronunciados nos níveis de glicose e insulina. Em contrapartida, as fibras solúveis como o $\beta$-glucano, psyllium e a goma-guar, em quantidades suficientes, diminuem os níveis de glicose e insulina pós-prandiais em indivíduos saudáveis. Efeitos benéficos após a segunda refeição a essa suplementação também têm sido descritos. Estudos com maior tempo de duração sobre os efeitos da aveia, psyllium e da goma-guar no metabolismo da glicose e insulina são poucos e não têm mostrado benefício algum. Por outro lado, estudos com o objetivo de medir somente o efeito das fibras em indivíduos onde esses benefícios seriam mais prováveis de ocorrer, como por exemplo em indivíduos com intolerância à glicose ou com síndrome metabólica, não têm sido conduzidos. $\mathrm{O}$ único estudo delineado para medir esses efeitos não demonstrou melhora na resposta da insulina de jejum. Ademais, os estudos devem ter uma duração de pelo menos dois meses. As metodologias utilizadas na determinação do metabolismo da glicose e insulina têm se restringido somente a medidas de glicose e insulina de jejum, não incluindo tanto o teste oral ou intravenoso de tolerância à glicose, ou ainda o clamp euglicêmico hiperinsulinêmico.

Em indivíduos obesos, por sua vez, apesar de alguns estudos de médio e longo prazos, com delineamento adequado, descreverem uma redução significativa do peso corporal pelo uso de suplementos de ricos em fibras solúveis, como o psyllium e a goma-guar, após uma vasta revisão de outras evidências na forma de metanálises, as evidências desses suplementos ricos em fibras na redução de peso não são conclusivas (18).

\section{Fibras da dieta e risco de diabetes melito tipo 2}

Não existem ainda estudos de intervenção que tenham focado no efeito isolado do consumo de fibras na mo- dificação do risco de desenvolver DM em indivíduos não diabéticos. No estudo finlandês de prevenção do diabetes (Diabetes Prevention Study: DPS), uma intervenção multifatorial que incluiu o aumento do consumo de fibras (principalmente fibras insolúveis) na dieta foi capaz de prevenir o desenvolvimento do DMT2 (2). Neste estudo, 523 indivíduos obesos com metabolismo anormal de glicose foram randomizados aos grupos de intervenção ou controle. Durante os três anos de estudo a redução do DM foi de $58 \%$. Quando os resultados foram analisados de acordo com o escore de sucesso à intervenção, aqueles indivíduos que alcançaram de quatro a cinco das metas estabelecidas (redução de peso mínima de $5 \%$, pelo menos quatro horas por semana de atividade física, consumo de fibras $>15 \mathrm{~g} / 1000 \mathrm{kcal}$, consumo de gorduras $<30 \%$ e desta $<10 \%$ de gordura saturada da energia total consumida), em ambos os grupos, não desenvolveram DM. Um estudo mais detalhado da dieta dos participantes deste estudo, no entanto, mostrou que a gordura da dieta - principalmente a gordura saturada, e o conteúdo de fibras consumido foram associados com a perda de peso contínua durante o estudo e a menor progressão para o DMT2, mesmo após ajustes para outros fatores de risco (3).

\section{Estudos epidemiológicos}

Com base em estudos epidemiológicos, a melhor evidência para o papel das fibras da dieta na prevenção do DMT2 ironicamente aponta para as fibras insolúveis. Dois grandes estudos de coorte realizados nos Estados Unidos, um deles com mais de 70.000 mulheres (47) e outro com mais de 42.000 homens (48), mostraram que as fibras contidas nos cereais, mas não aquelas provenientes das frutas ou das fibras solúveis, estavam associadas a uma diminuição do risco de DM em cerca de $30 \%$ quando feita a comparação entre os tercis de maior e menor valor levando em conta outros fatores de confusão e carga glicêmica da dieta. Outro estudo, publicado três anos adiante, confirmou esses achados em uma população de 36.000 mulheres americanas (49).

Os mecanismos pelos quais as fibras dos cereais, nestes estudos de coorte contendo apenas pouca quantidade de fibras solúveis como o $\beta$-glucano e o arabinoxilano, podem prevenir o DM de forma independente da carga glicêmica e peso corporal não são claros, uma vez que as fibras insolúveis, pelo menos a curto prazo, não têm efeito sobre o metabolismo da glicose e insulina. No entanto, a maioria dos prováveis efeitos protetores 
dos alimentos compostos de grãos integrais contra o DM parece ser mediada de fato pelo conteúdo de fibras dos cereais ou por componentes fortemente associados a estas $(49,50)$. É possível, porém, que essa associação seja em razão da presença de outros componentes dos grãos integrais. Em estudo prospectivo recente com cerca de 160.000 participantes cujos resultados foram compilados a quatro desses estudos de coorte citados em uma revisão sistemática $(51)$, foi demonstrado que o consumo de duas porções por dia de grãos integrais a mais na dieta estava associado a uma diminuição de $21 \%$ no risco de DM. No entanto, evidências epidemiológicas ainda não são suficientes para demonstrar o efeito protetor das fibras solúveis no desenvolvimento do DM. Isso se deve, talvez, ao fato de que uma grande quantidade de fibras solúveis na dieta seria necessária para alterar os seus efeitos nas respostas glicêmica e insulínica pós-prandiais. Essa quantidade de fibra que deveria ser aumentada na dieta, no entanto, é muito maior do que a quantidade que normalmente se consome na dieta ocidental. Ademais, a imprecisão dos dados provenientes da avaliação da dieta em estudos epidemiológicos é uma limitação.

\section{Fibras da dieta no tratamento da hiperglicemia no diabetes melito tipo 2}

Fibras solúveis, como o $\beta$-glucano da aveia, psyllium e a goma-guar, têm sido recomendadas a pacientes com DMT2 a fim de melhorar a resposta pós-prandial da insulina e glicose, além do seu efeito anti-hiperlipidêmico. É descrito na literatura e bem conhecido cientificamente o fato de que os polissacarídeos viscosos da dieta diminuem a taxa de absorção da digestão e a absorção dos carboidratos. Uma das principais razões pelas quais essas respostas estão mais lentas é o atraso na mistura do conteúdo no lúmen intestinal, que causa retardo na difusão e no contato entre as enzimas intestinais e os seus respectivos substratos, e retardo no transporte (52). Podemos encontrar na literatura estudo que mostra uma associação linear inversa entre os níveis pós-prandiais de glicose e insulina e o nível de viscosidade da mistura de líquidos ingeridos (29). A importância da viscosidade sobre a resposta glicêmica já tinha sido enfatizada em 1978 por Jenkins e cols. (53). As fibras insolúveis, por sua vez, não têm efeitos sobre o metabolismo da glicose e insulina, pelo menos a curto ou médio prazos (54), apesar de as evidências epidemiológicas referirem que essas fibras previnem o aparecimento do DM de forma independente à carga glicêmica ou ao IMC.

\section{Estudos pós-prandiais}

O $\beta$-glucano da aveia é conhecido pelo seu efeito na redução dos níveis pós-prandiais de glicose e insulina após carga oral glicêmica em pacientes diabéticos $(28,55,56)$. Tanto a goma isolada da aveia $(28)$ assim como o farelo da aveia que contém o $\beta$-glucano $(55,56)$ têm se mostrado benéficos. Tappy e cols. (55) demonstraram uma diminuição progressiva das concentrações plasmáticas de glicose de 33\% a $63 \%$ e das concentrações de insulina de $33 \%$ a $41 \%$ com a adição de 4 a $8,4 \mathrm{~g}$ de $\beta$-glucano ao café da manhã teste à base de farelo de aveia em comparação a um café da manhã controle. No entanto, os benefícios são dependentes da viscosidade da fibra e podem ser perdidos se esta vier a ser reduzida na consequência do processamento do alimento. A viscosidade do $\beta$-glucano, medida por uma técnica de simulação das condições fisiológicas, tem sido enfatizada também nos casos dos cereais matinais que contêm farelo de aveia na sua composição ou mistura (55). Tem sido discutido e firmado por alguns autores que predizer a ação dos polissacarídeos com base na sua viscosidade de pré-ingestão pode ser ilusória e que a viscosidade a ser considerada deve ser aquela no local de absorção no estômago (57).

O psyllium $(58,59)$ e a goma-guar $(60-62)$ diminuem as concentrações de insulina e glicose pós-prandiais em pacientes com DMT2. Em alguns estudos, no entanto, o efeito da goma-guar nos níveis pós-prandiais de glicose não tem sido demonstrado, até mesmo quando utilizadas doses de $5 \mathrm{~g}$ nas refeições (63).

\section{Estudos de médio e longo prazos}

Estudos de médio e longo prazos que medem o efeito das fibras solúveis da dieta na melhora do controle glicêmico no DM e que não utilizam a goma-guar não são muito comuns. Entre essas poucas intervenções está um estudo com homens diabéticos e hipercolesterolêmicos, em que estes foram randomizados a um grupo controle (placebo-celulose) ou grupo experimental (psyllium $10 \mathrm{~g} /$ dia) por oito semanas. No grupo experimental, a adição de psyllium foi capaz de reduzir a hemoglobina glicada $\left(\mathrm{HbA}_{1 c}\right)$ em 6,1\% (diferença absoluta de 0,8\%), com similar $6 \%$ de redução na concentração na glicose plasmática de jejum (64). No final do estudo, em relação ao grupo controle, houve também um efeito sobre a segunda refeição com uma expressiva diminuição na concentração de glicose pós-prandial em 11\% e 19\% após o almoço e no final do dia. No entanto, não foi observada mudança na sensibilidade à insulina medida por meio do método clamp euglicêmico hiperinsulinêmico. 
Vários estudos randomizados em pacientes com DMT2 foram conduzidos. Uma melhora no controle glicêmico pós-prandial e de jejum foi observada em 11 pacientes que fizeram uso de $21 \mathrm{~g} /$ dia de gomaguar comparado ao placebo em um estudo duplo-cego cruzado (65). Uma sensível melhora geral na resposta glicêmica e uma melhora expressiva na glicemia pósprandial após quatro semanas de suplementação com goma-guar em outro estudo do tipo cruzado foram descritas por Fuessl e cols. (61). Em um estudo também duplo-cego cruzado a goma-guar foi capaz de diminuir a concentração plasmática de glicose de 205 para $170 \mathrm{mg} / \mathrm{dl}(1 \mathrm{l}, 4$ to $9,5 \mathrm{mmol} / \mathrm{l})$ em 19 pacientes obesos com DMT2 (66). A goma-guar administrada a $15 \mathrm{~g}$ /dia por um período de oito semanas também resultou em efeitos benéficos a longo prazo sobre o controle glicêmico e intolerância à glicose em 15 pacientes com DMT2 (67). Por outro lado, a ingestão dessa mesma dose de goma-guar por três meses não mostrou efeito algum nos níveis de glicose sérica ou $\mathrm{HbA}_{\mathrm{lc}} \mathrm{em}$ pacientes que faziam uso de hipoglicemiante oral (68), assim como também em outro estudo cruzado com duração de oito semanas em pacientes tratados com dieta, hipoglicemiante oral ou insulina lenta (63).

Em conclusão, as fibras solúveis como o $\beta$-glucano, psyllium e goma-guar diminuem a resposta da insulina e da glicose aos carboidratos da dieta se administradas em doses suficientes. Efeitos benéficos após a segunda refeição a essa suplementação também têm sido descritos.

Em alguns estudos isolados que mediram o efeito do $\beta$-glucano e do psyllium por períodos que variaram de semanas a meses, foi sugerido um efeito benéfico no controle glicêmico a longo prazo. Porém, mais estudos são necessários para solidificar essa resposta glicêmica favorável. Resultados ainda são conflitantes em relação ao mais estudado tipo de fibra, a goma-guar.

\section{Direções futuras}

\section{Uso de fibras dietéticas}

Como descrito claramente nos capítulos anteriores neste artigo, as fibras dietéticas são de importância na manipulação de alimentos a fim de auxiliarem no melhor controle glicêmico. O conteúdo de fibras dietéticas no alimento pode ser aumentado tanto pelo uso de matéria-prima naturalmente rica em fibras quanto pelo uso de preparações comerciais de fibras adicionadas como ingredientes na sua confecção. Considerando a diver- sidade nos vários tipos de carboidratos ricos em fibras disponíveis, obviamente um cuidado especial e muitas vezes laborioso é necessário para atingir as propriedades fisiológicas desejadas para o alimento a ser produzido. Dessa forma, esse ramo industrial é desafiante, porém com alto potencial de benefício à saúde.

\section{Estudos de expressão gênica e metabolômica}

Estudos recentes, que utilizaram a técnica de microarrasto para estudos de expressão gênica (transcriptômica), mostraram que modificações da dieta podem promover uma modulação de dezenas ou até de centenas de genes, por exemplo, no tecido adiposo (69). O estudo de rotas metabólicas envolvendo os genes estudados pode trazer mais informações sobre a relevância biológica das diferenças observadas na expressão gênica a partir da modificação da dieta. A metabolômica, por sua vez, é uma ferramenta que utiliza técnicas e métodos estatísticos mais complexos para identificação de modulações em nível metabólico como, por exemplo, de compostos metabólicos lipídicos e proteicos. Com o auxílio dessas ferramentas estatísticas, a metabolômica auxilia no estudo de como as modulações na expressão dos genes em diferentes rotas metabólicas se relacionam a diferentes desfechos, como aqueles ligados ao metabolismo de insulina e glicose. A partir de tais técnicas, pode se esperar também informações adicionais sobre os mecanismos pelos quais as fibras dietéticas influenciam o metabolismo insulínico e glicídico.

\section{CONCLUSÕES}

Evidências convincentes, a partir de estudos epidemiológicos, suportam um papel para os produtos alimentícios à base de grãos integrais e para as fontes de fibras insolúveis na prevenção do DMT2. Paradoxalmente, em estudos pós-prandiais, as fibras solúveis e não as insolúveis é que promovem um efeito favorável no metabolismo da glicose e da insulina, se administradas em quantidades suficientes. Resultados de estudos de médio e longo prazos sobre o papel das fibras dietéticas na melhora do metabolismo glicídico e insulínico, tanto em indivíduos com ou sem DM, são menos conclusivos. Um maior número de ensaios clínicos randomizados é necessário para se poder estabelecer o papel das fibras dietéticas na prevenção e tratamento do DMT2. Parece claro, no entanto, que se está somente no começo do real entendimento sobre a im- 
portância da qualidade dessas fibras dietéticas. Existem vários fatores do próprio alimento que influenciam a funcionalidade fisiológica dos carboidratos presentes nos produtos alimentícios além do conteúdo de fibras. No futuro, novas tecnologias nos ramos das técnicas de transcriptômica e matabolômica poderão nos trazer uma percepção mais abrangente dos efeitos fisiológicos das fibras dietéticas.

Agradecimentos: à Fundação Cultural da Finlândia, Fundos da Região Norte de Savo (Suomen Kulttuurirahasto, PohjoisSavon rahaston) pelo auxílio financeiro à Dra. Vanessa D. de Mello durante a redação deste manuscrito.

Declaração: os autores declaram não haver conflito de interesse científico neste artigo.

\section{BIBLIOGRAFIA}

1. American Diabetes Association. Standards of Medical Care in Diabetes - 2009 10.2337/dc09-S013. Diabetes Care. 2009;32 (Suppl1):S13-61.

2. Tuomilehto J, Lindström J, Eriksson JG, Valle TT, Hämäläinen $H$, llanne-Parikka $\mathrm{P}$, et al. Prevention of type 2 diabetes mellitus by changes in lifestyle among subjects with impaired glucose tolerance. N Engl J Med. 2001;344(18):1343-50.

3. Lindström J, Peltonen M, Eriksson JG, Louheranta A, Fogelholm M, Uusitupa $M$, et al. High-fibre, low-fat diet predicts long-term weight loss and decreased type 2 diabetes risk: the Finnish Diabetes Prevention Study. Diabetologia 2006;49(5):912-20.

4. Executive Summary of TheThird Report ofThe National Cholesterol Education Program (NCEP) Expert Panel on Detection, Evaluation, And Treatment of High Blood Cholesterol In Adults (Adult Treatment Panel III). JAMA. 2001;285(19):2486-97.

5. Alberti KG, Zimmet $P$, Shaw J. Metabolic syndrome - a new world-wide definition. A Consensus Statement from the International Diabetes Federation. Diabet Med. 2006;23(5):469-80.

6. Reaven GM. Banting lecture 1988. Role of insulin resistance in human disease. Diabetes. 1988;37(12):1595-607.

7. Laaksonen DE, Lakka HM, Niskanen LK, Kaplan GA, Salonen JT, Lakka TA. Metabolic syndrome and development of diabetes mellitus: application and validation of recently suggested definitions of the metabolic syndrome in a prospective cohort study. Am J Epidemiol. 2002;156(11):1070-7.

8. Lakka HM, Laaksonen DE, Lakka TA, Niskanen LK, Kumpusalo $\mathrm{E}$, Tuomilehto $\mathrm{J}$, et al. The metabolic syndrome and total and cardiovascular disease mortality in middle-aged men. JAMA. 2002;288(21):2709-16.

9. Kahn SE. The relative contributions of insulin resistance and betacell dysfunction to the pathophysiology of Type 2 diabetes. Diabetologia. 2003;46(1):3-19.

10. Edelstein SL, Knowler WC, Bain RP, Andres R, Barrett-Connor EL, Dowse GK, et al. Predictors of progression from impaired glucose tolerance to NIDDM: an analysis of six prospective studies. Diabetes. 1997;46(4):701-10.

11. DeVries JW. On defining dietary fibre. Proc Nutr Soc. 2003;62(1):37-43.

12. Cummings $\mathrm{JH}$, Mann JI, Nishida C, Vorster HH. Dietary fibre: an agreed definition. Lancet. 2009;373(9661):365-6.

13. Suter PM. Carbohydrates and dietary fiber. Handb Exp Pharmacol. 2005(170):231-61.

14. Diet, nutrition and the prevention of chronic diseases. World Health Organ Tech Rep Ser. 2003;916:i-viii, 1-149, backcover.
15. Liu S, WillettWC, Manson JE, Hu FB, Rosner B, Colditz G. Relation between changes in intakes of dietary fiber and grain products and changes in weight and development of obesity among middle-aged women. Am J Clin Nutr. 2003;78(5):920-7.

16. Savage JS, Marini M, Birch LL. Dietary energy density predicts women's weight change over 6 y. Am J Clin Nutr. 2008;88(3):677-84.

17. Vioque J, Weinbrenner T, Castello A, Asensio L, Garcia de la Hera M. Intake of fruits and vegetables in relation to 10 -year weight gain among Spanish adults. Obesity (Silver Spring). 2008;16(3):664-70.

18. Galisteo M, Duarte J, Zarzuelo A. Effects of dietary fibers on disturbances clustered in the metabolic syndrome. J Nutr Biochem. 2008;19(2):71-84

19. Kromhout D, Bloemberg B, Seidell JC, Nissinen A, Menotti A Physical activity and dietary fiber determine population body fat levels: the Seven Countries Study. Int J Obes Relat Metab Disord. 2001;25(3):301-6.

20. Ludwig DS, Pereira MA, Kroenke $\mathrm{CH}$, Hilner JE, Van Horn L, Slattery $\mathrm{ML}$, et al. Dietary fiber, weight gain, and cardiovascular disease risk factors in young adults. JAMA. 1999;282(16):1539-46.

21. Koh-Banerjee P, Franz M, Sampson L, Liu S, Jacobs DR Jr., Spiegelman $D$, et al. Changes in whole-grain, bran, and cereal fiber consumption in relation to $8-y$ weight gain among men. Am J Clin Nutr. 2004;80(5):1237-45.

22. Good CK, Holschuh N, Albertson AM, Eldridge AL. Whole grain consumption and body mass index in adult women: an analysis of NHANES 1999-2000 and the USDA Pyramid Servings Database. J Am Coll Nutr. 2008;27(1):80-7.

23. Harland JI, Garton LE. Whole-grain intake as a marker of healthy body weight and adiposity. Public Health Nutr. 2008;11(6):554-63.

24. McKeown NM, Meigs JB, Liu S, Saltzman E, Wilson PW, Jacques PF. Carbohydrate nutrition, insulin resistance, and the prevalence of the metabolic syndrome in the Framingham Offspring Cohort. Diabetes Care. 2004;27(2):538-46.

25. Ylonen K, Saloranta C, Kronberg-Kippila C, Groop L, Aro A, Virtanen SM. Associations of dietary fiber with glucose metabolism in nondiabetic relatives of subjects with type 2 diabetes: the Botnia Dietary Study. Diabetes Care. 2003;26(7):1979-85.

26. Sahyoun NR, Jacques PF, Zhang XL, JuanW, McKeown NM. Wholegrain intake is inversely associated with the metabolic syndrome and mortality in older adults. Am J Clin Nutr. 2006;83(1):124-31.

27. Steemburgo T, Dall'Alba V, Almeida JC, Zelmanovitz T, Gross JL, de Azevedo MJ. Intake of soluble fibers has a protective role for the presence of metabolic syndrome in patients with type 2 diabetes. Eur J Clin Nutr. 2009;63(1):127-33.

28. Braaten JT, Wood PJ, Scott FW, Riedel KD, Poste LM, Collins MW. Oat gum lowers glucose and insulin after an oral glucose load. Am J Clin Nutr. 1991;53(6):1425-30.

29. Wood PJ, Braaten JT, Scott FW, Riedel KD, Wolynetz MS, Collins MW. Effect of dose and modification of viscous properties of oat gum on plasma glucose and insulin following an oral glucose load. Br J Nutr. 1994;72(5):731-43.

30. Rigaud D, Paycha F, Meulemans A, Merrouche M, Mignon M. Effect of psyllium on gastric emptying, hunger feeling and food intake in normal volunteers: a double blind study. Eur J Clin Nutr. 1998;52(4):239-45.

31. Frape DL, Jones AM. Chronic and postprandial responses of plasma insulin, glucose and lipids in volunteers given dietary fibre supplements. Br J Nutr. 1995;73(5):733-51.

32. Jarjis HA, Blackburn NA, Redfern JS, Read NW. The effect of ispaghula (Fybogel and Metamucil) and guar gum on glucose tolerance in man. Br J Nutr. 1984;51(3):371-8.

33. Leclère CJ, Champ M, Boillot J, Guille G, Lecannu G, Molis C, et al. Role of viscous guar gums in lowering the glycemic response after a solid meal. Am J Clin Nutr. 1994;59(4):914-21. 
34. Ellis PR, Dawoud FM, Morris ER. Blood glucose, plasma insulin and sensory responses to guar-containing wheat breads: effects of molecular weight and particle size of guar gum. $\mathrm{Br} \mathrm{J}$ Nutr. 1991;66(3):363-79.

35. Bjorck I, Elmstahl HL. The glycaemic index: importance of dietary fibre and other food properties. Proc Nutr Soc. 2003;62(1):201-6.

36. Liljeberg HG, Akerberg AK, Bjorck IM. Effect of the glycemic index and content of indigestible carbohydrates of cereal-based breakfast meals on glucose tolerance at lunch in healthy subjects. Am J Clin Nutr. 1999;69(4):647-55.

37. Davy BM, Davy KP, Ho RC, Beske SD, Davrath LR, Melby CL. High-fiber oat cereal compared with wheat cereal consumption favorably alters LDL-cholesterol subclass and particle numbers in middle-aged and older men. Am J Clin Nutr. 2002;76(2):351-8.

38. Keogh GF, Cooper GJ, MulveyTB, McArdle BH, Coles GD, Monro $\mathrm{JA}$, et al. Randomized controlled crossover study of the effect of a highly beta-glucan-enriched barley on cardiovascular disease risk factors in mildly hypercholesterolemic men. Am J Clin Nutr. 2003;78(4):711-8.

39. Kirby RW, Anderson JW, Sieling B, Rees ED, Chen WJ, Miller RE, et al. Oat-bran intake selectively lowers serum low-density lipoprotein cholesterol concentrations of hypercholesterolemic men. Am J Clin Nutr. 1981;34(5):824-9.

40. Uusitupa MI, Ruuskanen E, Makinen E, Laitinen J, Toskala E, Kervinen $\mathrm{K}$, et al. A controlled study on the effect of beta-glucan-rich oat bran on serum lipids in hypercholesterolemic subjects: relation to apolipoprotein E phenotype. J Am Coll Nutr. 1992;11(6):651-9.

41. Anderson JW, Zettwoch N, Feldman T, Tietyen-Clark J, Oeltgen $\mathrm{P}$, Bishop CW. Cholesterol-lowering effects of psyllium hydrophilic mucilloid for hypercholesterolemic men. Arch Intern Med. 1988;148(2):292-6.

42. Bell LP, Hectorne K, Reynolds H, Balm TK, Hunninghake DB. Cholesterol-lowering effects of psyllium hydrophilic mucilloid. Adjunct therapy to a prudent diet for patients with mild to moderate hypercholesterolemia. JAMA. 1989;261(23):3419-23.

43. Katcher HI, Legro RS, Kunselman AR, Gillies PJ, Demers LM, Bagshaw DM, et al. The effects of a whole grain-enriched hypocaloric diet on cardiovascular disease risk factors in men and women with metabolic syndrome. Am J Clin Nutr. 2008;87(1):79-90.

44. Garcia AL, Steiniger J, Reich SC, Weickert MO, Harsch I, Machowetz $A$, et al. Arabinoxylan fibre consumption improved glucose metabolism, but did not affect serum adipokines in subjects with impaired glucose tolerance. Horm Metab Res. 2006;38(11):761-6.

45. Laaksonen DE, Toppinen LK, Juntunen KS, Autio K, Liukkonen KH, Poutanen KS, et al. Dietary carbohydrate modification enhances insulin secretion in persons with the metabolic syndrome. Am J Clin Nutr. 2005;82(6):1218-27.

46. Juntunen KS, Laaksonen DE, Autio K, Niskanen LK, Holst JJ, Savolainen $\mathrm{KE}$, et al. Structural differences between rye and wheat breads but not total fiber content may explain the lower postprandial insulin response to rye bread. Am J Clin Nutr. 2003;78(5):957-64.

47. Salmeron J, Manson JE, Stampfer MJ, Colditz GA, Wing AL, Willett WC. Dietary fiber, glycemic load, and risk of non-insulin-dependent diabetes mellitus in women. JAMA. 1997;277(6):472-7.

48. Salmeron J, Ascherio A, Rimm EB, Colditz GA, Spiegelman D, Jenkins DJ, et al. Dietary fiber, glycemic load, and risk of NIDDM in men. Diabetes Care. 1997;20(4):545-50.

49. Meyer KA, Kushi LH, Jacobs DR Jr, Slavin J, Sellers TA, Folsom AR. Carbohydrates, dietary fiber, and incident type 2 diabetes in older women. Am J Clin Nutr. 2000;71(4):921-30.

50. Montonen J, Knekt P, Jarvinen R, Aromaa A, Reunanen A. Wholegrain and fiber intake and the incidence of type 2 diabetes. Am J Clin Nutr. 2003;77(3):622-9.

51. de Munter JS, Hu FB, Spiegelman D, Franz M, van Dam RM. Whole grain, bran, and germ intake and risk of type 2 diabetes: a prospective cohort study and systematic review. PLoS Med. 2007;4(8):e261.

52. Edwards CA, Johnson IT, Read NW. Do viscous polysaccharides slow absorption by inhibiting diffusion or convection? Eur J Clin Nutr. 1988;42(4):307-12.

53. Jenkins DJ, WoleverTM, Leeds AR, Gassull MA, Haisman P, Dilawari J, et al. Dietary fibres, fibre analogues, and glucose tolerance: importance of viscosity. Br Med J. 1978;1(6124):1392-4.

54. Fontvieille AM, Bornet F, Rizkalla SW, Le Francois P, Pichard P, Desplanque $\mathrm{N}$, et al. In vitro and in vivo digestibility and metabolic effects of 3 wheat-flour products (white bread, french toast (rusk) and french toast bran-enriched) in normal subjects. Diabete Metab. 1988;14(2):92-6.

55. Tappy L, Gugolz E, Wursch P. Effects of breakfast cereals containing various amounts of beta-glucan fibers on plasma glucose and insulin responses in NIDDM subjects. Diabetes Care. 1996;19(8):831-4.

56. Tapola N, Karvonen H, Niskanen L, Mikola M, Sarkkinen E. Glycemic responses of oat bran products in type 2 diabetic patients. Nutr Metab Cardiovasc Dis. 2005;15(4):255-61.

57. Edwards CA, Blackburn NA, Craigen L, Davison P, Tomlin J, Sugden $\mathrm{K}$, et al. Viscosity of food gums determined in vitro related to their hypoglycemic actions. Am J Clin Nutr. 1987;46(1):72-7.

58. Pastors JG, Blaisdell PW, Balm TK, Asplin CM, PohI SL. Psyllium fiber reduces rise in postprandial glucose and insulin concentrations in patients with non-insulin-dependent diabetes. Am J Clin Nutr. 1991;53(6):1431-5.

59. Rodriguez-Moran M, Guerrero-Romero F, Lazcano-Burciaga G. Lipid- and glucose-lowering efficacy of Plantago Psyllium in type II diabetes. J Diabetes Complications. 1998;12(5):273-8.

60. Jenkins DJ, Leeds AR, Gassull MA, Cochet B, Alberti GM. Decrease in postprandial insulin and glucose concentrations by guar and pectin. Ann Intern Med. 1977;86(1):20-3.

61. Fuessl HS, Williams G, Adrian TE, Bloom SR. Guar sprinkled on food: effect on glycaemic control, plasma lipids and gut hormones in non-insulin dependent diabetic patients. Diabet Med. 1987:4(5):463-8.

62. Gatenby SJ, Ellis PR, Morgan LM, Judd PA. Effect of partially depolymerized guar gum on acute metabolic variables in patients with non-insulin-dependent diabetes. Diabet Med. 1996;13(4):358-64.

63. Holman RR, Steemson J, Darling P, Turner RC. No glycemic benefit from guar administration in NIDDM. Diabetes Care. 1987;10(1):68-71.

64. Anderson JW, Allgood LD, Turner J, Oeltgen PR, Daggy BP. Effects of psyllium on glucose and serum lipid responses in men with type 2 diabetes and hypercholesterolemia. Am J Clin Nutr. 1999;70(4):466-73.

65. Aro A, Uusitupa M, Voutilainen E, Hersio K, Korhonen T, Siitonen O. Improved diabetic control and hypocholesterolaemic effect induced by long-term dietary supplementation with guar gum in type 2 (insulin-independent) diabetes. Diabetologia. 1981;21(1):29-33.

66. Lalor $\mathrm{BC}$, Bhatnagar D, Winocour $\mathrm{PH}$, Ishola $\mathrm{M}$, Arrol S, Brading $\mathrm{M}$, et al. Placebo-controlled trial of the effects of guar gum and metformin on fasting blood glucose and serum lipids in obese, type 2 diabetic patients. Diabet Med. 1990;7(3):242-5.

67. Groop PH, Aro A, Stenman S, Groop L. Long-term effects of guar gum in subjects with non-insulin-dependent diabetes mellitus. Am J Clin Nutr. 1993;58(4):513-8.

68. Uusitupa M, Siitonen O, Savolainen K, Silvasti M, Penttila I, Parviainen M. Metabolic and nutritional effects of long-term use of guar gum in the treatment of noninsulin-dependent diabetes of poor metabolic control. Am J Clin Nutr. 1989;49(2):345-51.

69. Kallio P, Kolehmainen M, Laaksonen DE, Kekalainen J, Salopuro $\mathrm{T}$, Sivenius K, et al. Dietary carbohydrate modification induces alterations in gene expression in abdominal subcutaneous adipose tissue in persons with the metabolic syndrome: the FUNGENUT Study. Am J Clin Nutr. 2007;85(5):1417-27. 